\title{
Steady-state ion pumping of a potential dip near an electron collecting anode
}

\author{
Cary Forest and Noah Hershkowitz \\ Nuclear Engineering Department, University of Wisconsin-Madison, Madison, Wisconsin 53706
}

(Received 8 January 1986; accepted for publication 21 April 1986)

\begin{abstract}
A negative potential well is found in front of a positively biased electron collecting plate in a low temperature laboratory plasma. The well parameters are governed by the Child-Langmuir condition and a necessary condition for the existence of such potential wells is the presence of an ion pumping mechanism which removes trapped ions from the wells. Here ion pumping was provided by the presence of negative electrically floating ceramic on the back of the plate.
\end{abstract}

\section{INTRODUCTION}

Potential dips separating regions with two different plasma potentials are a general feature of many different plasmas. These include positively biased probes in plasmas, ${ }^{1}$ multiple double layers in laboratory plasmas, ${ }^{2-5}$ ion acoustic double layers in space plasmas ${ }^{6.7}$ and in computer simulations, ${ }^{8}$ and thermal barriers in tandem mirrors. ${ }^{9,10}$ These structures have the common feature that the potential wells tend to fill up with ions, which either scatter into the potential well or are born by charge exchange or ionization within the well, and so they should cease to exist. Nevertheless, stationary structures have been produced or found in tandem mirrors, ${ }^{11}$ near objects inserted into plasmas, ${ }^{1}$ near cathodes, ${ }^{12.13}$ and at magnetic constrictions in magnetized $Q$ machine plasmas. ${ }^{14}$ All of these structures must provide some form of "pumping"- the removal of ions which continuously trap within the wells. Of the variety of situations in which stationary potential dips are known, thermal barriers are the only one for which the necessity for pumping has been recognized. Without pumping, stationary potential dips are not self-consistent structures. Nonstationary structures with potential dips are also known but these can be selfconsistent. $^{15}$

Thermal barriers ${ }^{9,10}$ are potential dips which provide electron thermal insulation between central cell and end cell electrons in tandem mirrors. This allows end cell electrons to be heated to higher temperatures and results in large ion confining potentials. Barrier pumping has been successfully accomplished in two tandem mirror experiments ${ }^{10.11}$ by using charge exchange on energetic neutral beams injected into the end cells. An alternative technique, rf drift pumping, ${ }^{16}$ has also been proposed for barrier pumping. Recently it has been demonstrated that ICRF ( $\mathrm{ff}$ near the ion cyclotron frequency) can provide electron pumping ${ }^{17}$ resulting in steady-state potential peaks. Potential peaks have also been achieved by increasing plasma density by adding ions to localized regions, e.g., neutral beam injection into the end cells of a tandem mirror. ${ }^{18}$ Ion injection represents a process which is the opposite of pumping.

Plasma potential modification by (ion or electron) pumping has now been demonstrated in many different situations. However many systems have been found which exhibit potential dips for which the pumping mechanism is not yet known. It is likely that a large variety of pumping mecha- nisms exist and it may be that the dips near probes, at magnetic constrictions, etc., all have different mechanisms. In this paper we investigate the process which provides ion pumping from the potential dip near a positively biased plate in a plasma.

\section{EXPERIMENTAL CONSIDERATHONS}

Data were taken in a $40-1$ stainless-steel soup pot with full line cusps ${ }^{19}$ consisting of 14 rows of permanent magnets on the grounded cylindrical walls and on one end. An argon plasma was produced by primary ionizing electrons emitted from tungsten filaments. The base pressure was $1 \times 10^{-6}$ Torr, and the data were taken with neutral pressures between $5 \times 10^{-5}$ and $8 \times 10^{-4}$ Torr. The density varied from $8 \times 10^{7}$ to $2 \times 10^{9} \mathrm{~cm}^{-3}$, with electron temperatures $T_{e}$ ranging from 2 to $4 \mathrm{eV}$ depending on the neutral pressure. Electron Debye lengths varied from 0.02 to $0.1 \mathrm{~cm}$.

Plasma potentials were measured with emissive probes using the inflection point technique. ${ }^{20}$ Density and electron temperature were measured using a $0.64-\mathrm{cm}$-diam Langmuir disk probe. Representative data are given in Fig. 1 which shows the plasma potential along the axis of a $7-\mathrm{cm}$ diam brass plate ( $2 \mathrm{~mm}$ thick); one side (the back) is covered by ceramic. The plate was biased at $+20 \mathrm{~V}$ with respect to ground, the back floats at $\sim-10 \mathrm{~V}$. It is apparent that the potential decreases from the uncoated surface of the plate to a potential minimum, passes through an inflection point, and then increases by $\sim T_{e} / 2 e$ to the potential far from the plate. $T_{e}$ is the ellectron temperature (equal to 3.5 $\mathrm{eV}$ for these data) and $e$ is the electron charge.

We have investigated the dependence on plasma parameters of the potential step $(\Delta \phi)$ between the background plasma and the potential dip, and the position of the dip ( $d$ ) with respect to the plate. Results are summarized in Fig. 2. The general features are that as the density increases the dip moves closer to the plate and that $T_{e}$ is proportional to $\Delta \phi$. For fixed plasma density, the separation distance $d$ does not appear to be a function of neutral pressure.

The understanding of the dip can be divided into two separate issues. One is the question of why the dip exists, i.e., the nature of the pumping, and the other is the physics associated with the dip itself. First we consider the pumping. Data which gave a hint to the nature of the pumping mechanism are shown in Fig. 3. A careful investigation of the equi- 


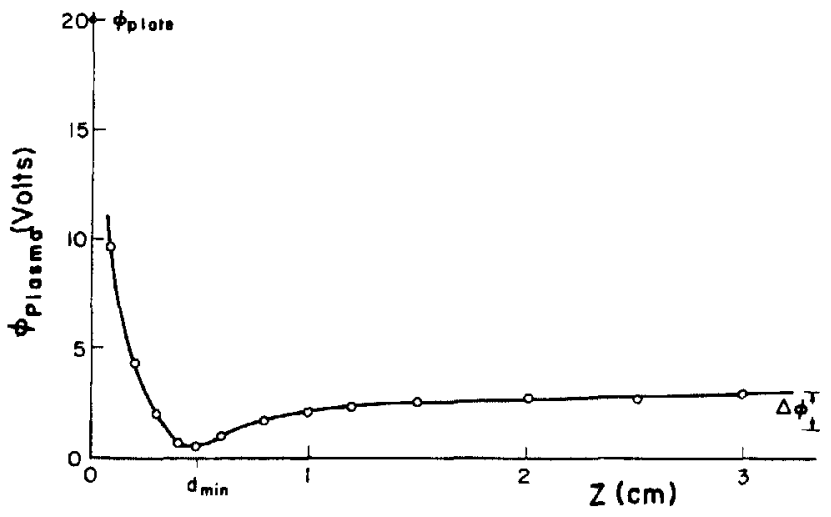

FIG. 1. The plasma potential, measured on axis of a circular plate biased at $+20 \mathrm{~V}$. The electron temperature was measured to be $T_{\mathrm{e}}=3.5 \mathrm{eV}$.

potential contours near a particular plate (coated on the back with ceramic) found a potential minimum (indicated by $+4 \mathrm{~V}$ ) attached to a particular position on the plate. The position of the minimum was not affected by external fields or plasma parameters. This dip was identified as being associated with a fingerprint on the plate. Ions can drain to the front of the plate because of the fingerprint's potential minimum.

The equipotential contours in the neighborhood of the biased plate (with the fingerprint cleaned off) are given in Fig. 4. Note that the potential dip in front of the plate is quite one dimensional in nature, consistent with the Debye length being much smaller than the plate radius. However, it is apparent that cold ions are not electrostatically trapped by the potential dip as ions can escape out of the edges of the channel formed by the dip because the potential becomes more negative there. These leaks provide the necessary pumping.

For the configuration shown in Fig. 4 the potential can
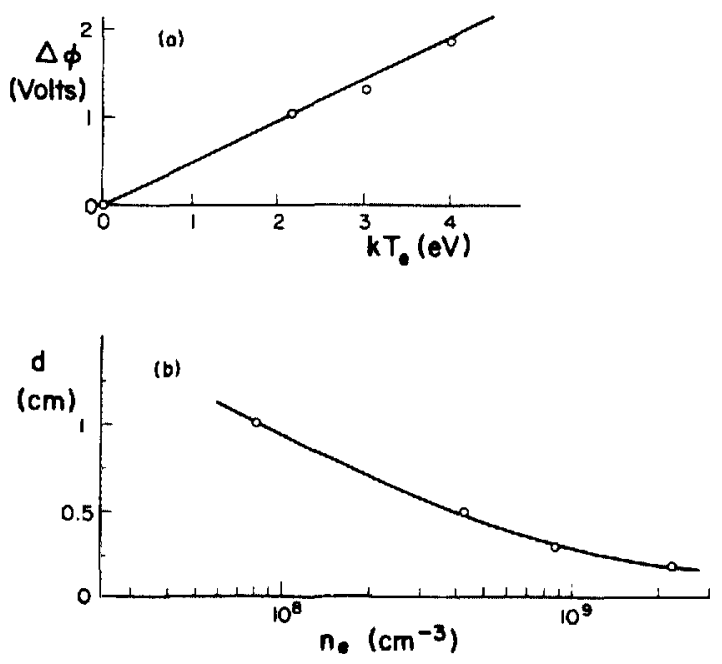

FIG. 2. (a) The potential difference between the plasma and the inflection point of the dip, as a function of electron temperature. A straight line is drawn through the data. (b) The penetration of the dip (d), as a function of plasma density. A smooth curve is drawn through the data.

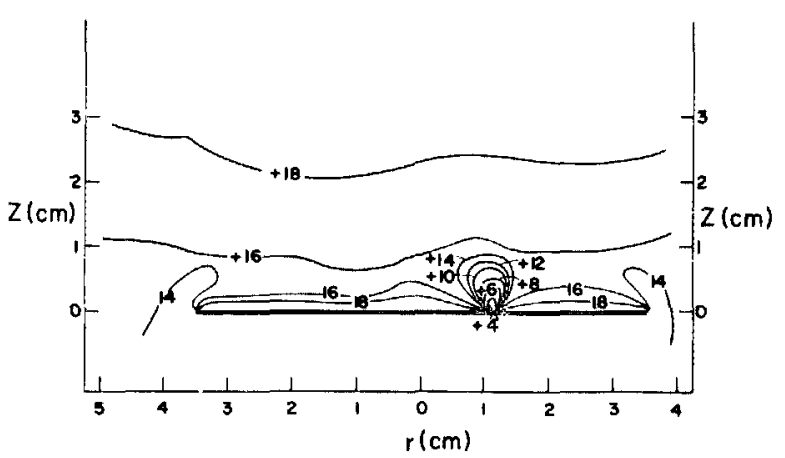

FIG. 3. The equipotential contours near a circular brass plate $(r=3.5 \mathrm{~cm}, 2$ $\mathrm{mm}$ thick). Note the contours near a grease fingerprint. The plate is in the center of the multidipole soup pot device, $10 \mathrm{~cm}$ from one end.

become more negative in the dip because of the presence of the floating insulator on the plate. We can demonstrate that this is a necessary condition for the dips existence by removing the insulator from the back of the plate. The potentials along the axis of the plate which correspond to this configuration are given in Fig. 5. In this case the plasma potential far from the plate becomes more positive than the potential on the plate and no dip is found. The plasma potential decreases monotonically to the plate. We varied the amount of ceramic on the back and found that dip only formed when the ceramic was within $1 \mathrm{~cm}$ or approximately 10 Debye lengths of the edge. It is only then that the negative potential contours are able to wrap around to the front of the plate giving a loss mechanism to the ions which would otherwise be trapped. Plasma ions which enter the dip need not be trapped. Ions which are created in the dip, however, have no way of escaping along the axis, and must therefore be lost radially to the ceramic.

The geometry of the plate and the ceramic is important. This was demonstrated by varying the location of the ceramic with respect to the plate. A ceramic disk the area of the plate ceramic was placed randomly in the volume of the plasma ( $\sim 10 \mathrm{~cm}$ from the uncoated plate). In this case the plasma potential far from the plate and the insulator was found. to be the same positive value as was found when no insulator was present.

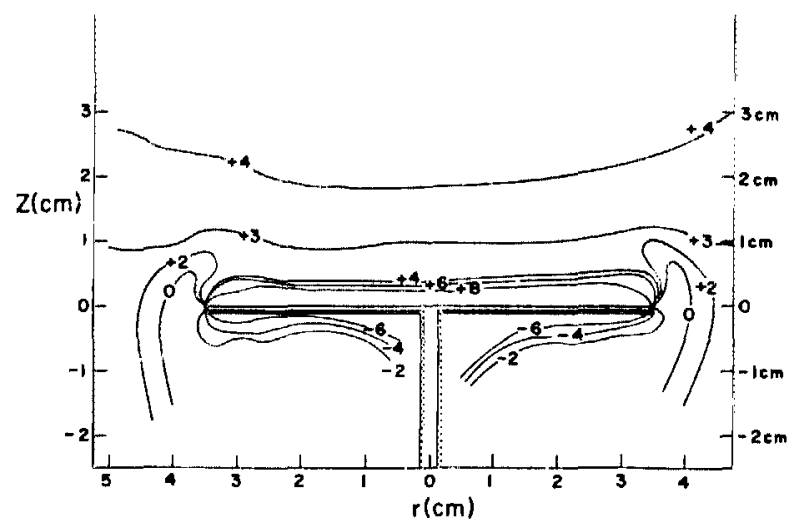

FIG. 4. The equipotential contours near a clean plate. The back of the plate, the edges of the plate, and the support are covered with ceramic. 


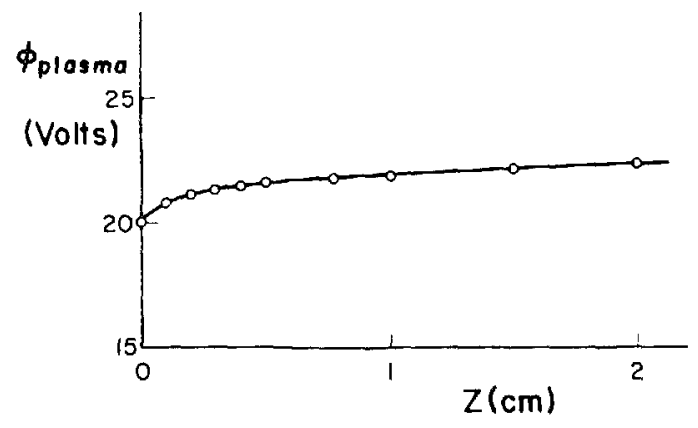

FIG. 5. The plasma potential measured on the axis of the plate with no ceramic on the back.

\section{DISCUSSION}

First we consider the physics of dip. As we approach the plate from the background plasma potential (see Fig. 1), a presheath is apparent which provides initial acceleration to ions. This structure is similar to the Bohm presheath ${ }^{21}$ associated with an ordinary monotonic sheath in that it has negative curvature. It differs from the Bohm presheath in that there is an inflection point (at the presheath edge) where the ion and electron densities are equal. At smaller $z$ the electron density exceeds the ion density which indicates ${ }^{21}$ that the presheath must accelerate ions to a velocity which is somewhat less than the ion acoustic velocity $c_{s}=\left(T_{e} / m_{i}\right)^{1 / 2}$. This corresponds to a presheath potential $\leqslant T_{e} / 2 e$. If the density of trapped particles is not too high and the deviation from quasineutrality is small, the equality sign holds. Note that the presheath potentials shown in Figs. 1 and 4 approximately satisfy $\Delta \phi=T_{e} / 2 e$.

For potentials near the positively biased plate, that are more positive than the background potential, only electrons are present (except for a small population of ions produced by ionization). We expect the potential to be space-charge limited there and that $d$ is determined by the Child-Langmuir condition. ${ }^{12}$ The electron current density which gets over the Bohm presheath potential $\Delta \phi$ is given by

$$
J=e n \sqrt{T_{e} / 2 \pi m_{e}} \exp \left(-e \Delta \phi / T_{e}\right),
$$

where $n$ is the plasma density. Assuming that the dip forms to give a Bohm velocity, we can write

$d_{\min }^{2}=1.02 \times 10^{6} \frac{\left(\phi_{\text {plate }}-\phi_{\mathrm{dip}}\right)^{3 / 2}}{n_{e} \sqrt{T_{e}}}\left(1+\frac{2.66}{\sqrt{e \phi_{\text {plate }} / T_{e}}}\right)$

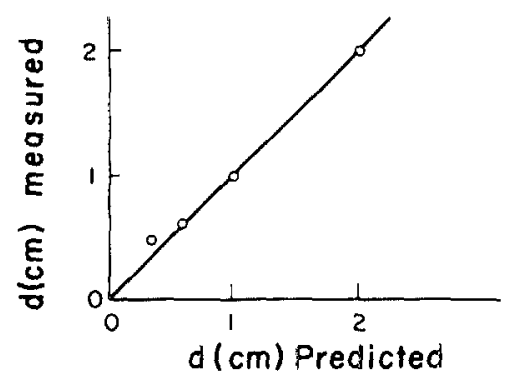

FIG. 6. A comparison between the predicted and the measured values of $d_{\min }$.
Experimental values of $d$ are compared to predictions from Eq. (2) in Fig. 6. It is apparent that there is good agreement between the data and the predictions of Eq. (2).

The plasma potential is determined by a balance of electron and ion creation and loss. By considering the losses to the plate, the effect of plate bias on the plasma potential can be found. The back of the plate is floating, and is therefore an equal loss for both species. There is no ion current to the front of the plate because of the large potential barrier. The electron current is simply the current density which gets over the $T_{e} / 2 e$ potential dip [Eq. (1)] $\times$ collecting area. Nowhere does the value of the plasma potential play a role, except in determining the effective collecting area of the plate. Evidence for this is given in Fig. 7 which shows that the plasma potential is unaffected by the plate voltage beyond $20 \mathrm{~V}$. The plate size is large enough that when the ceramic is not present the plate becomes the major electron loss, as shown in Fig. 5.

Removal of $1 \mathrm{~cm}$ of insulator at the circumference on the back of the plate also resulted in inadequate pumping. We can estimate the conditions which must be satisfied in order to provide adequate ion pumping as follows: trapped ion production by charge exchange and ionization must be less than ion leaks (i.e., pumping) out of the edges of the dip. This gives

$n_{i} \pi r^{2} W n_{0}\left[\sigma_{c x} c_{s}+\left(n_{p} / n_{i}\right) \sigma_{i} v_{p}\right] \leqslant 2 \pi r\left(W c_{s} / 2\right) n_{i}$,

where $W$ is the dip width, $c_{s}$ is the ion acoustic velocity, $n_{i}, n_{0}, n_{t}$, and $n_{p}$ are the background untrapped ion, neutral, trapped ion density, and ionizing electron density, respectively, $r$ is the plate radius, and $\sigma_{i}$ and $\sigma_{c x}$ are the cross sections for ionization ${ }^{22}$ and charge exchange, ${ }^{23}$ respectively. Equation (3) can be rewritten as a condition on the neutral density:

$$
n_{0} \leqslant \frac{c_{s}\left(n_{t} / n_{i}\right)}{r\left[\sigma_{c x} c_{s}+n_{p} /\left(n_{i} \sigma_{i} v_{p}\right)\right]} .
$$

We can estimate $n_{p} / n_{i}$ by balancing ion production with ion losses in the multidipole device. This gives

$$
n_{p} V \sigma_{i} n_{0} v_{p}=\frac{1}{2} n_{i} c_{s} A,
$$

where $V$ is the plasma volume and $A$ the ion loss area. Combining Eqs. (4) and (5) gives

$$
n_{0} \leqslant \frac{n_{t} / n_{i}}{r\left[\sigma_{c x}+A /\left(V 2 n_{0}\right)\right]} .
$$

At high neutral pressures Eq. (6) gives

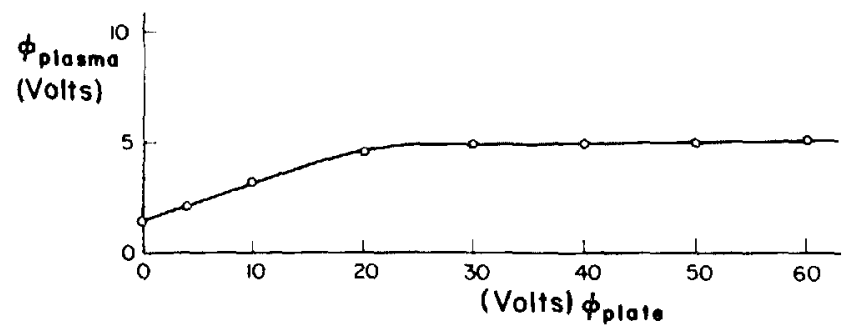

FIG. 7. The effect of the plate potential on the plasma potential. 


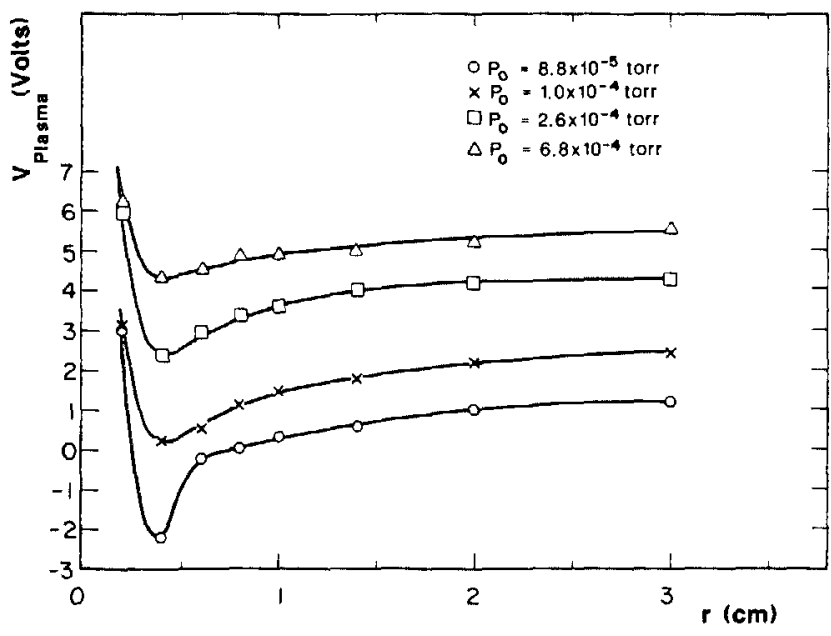

FIG. 8. The axial potential profiles of the disk shown in Fig. 3 at several neutral pressures.

$$
r n_{0} \leqslant\left(n_{t} / n_{i}\right) / \sigma_{c x}
$$

at low neutral pressures where $A /\left(V 2 n_{0}>\sigma_{c x}\right)$,

$$
r \leqslant\left(2 n_{t} / n_{i}\right) V / A \text {. }
$$

Figure 8 gives the plasma potential axial profile for several neutral pressures. It is apparent that the plasma potential far from the plate increases and the dip decreases as the neutral pressure is increased. Note that the position of the dip remains approximately constant. The plasma potential is determined by the balance of ion and electron production and loss and at lower neutral pressures, secondary electrons and primary ionizing electrons make an important contribution to determining the potential. From Eq. (5) it is apparent that the primary density is inversely proportional to the neutral pressure. Equation (7) shows that the potential dip cannot be established if the neutral pressure is sufficiently high since $n_{t} / n_{i}$ must be $<1$. Data for the plasma potential far from the plate are given in Fig. 9. At low neutral pressure the plasma potential is very negative with respect to the plate. As the neutral pressure is increased the potential becomes more positive and approaches the plate potential eliminating the potential dip.

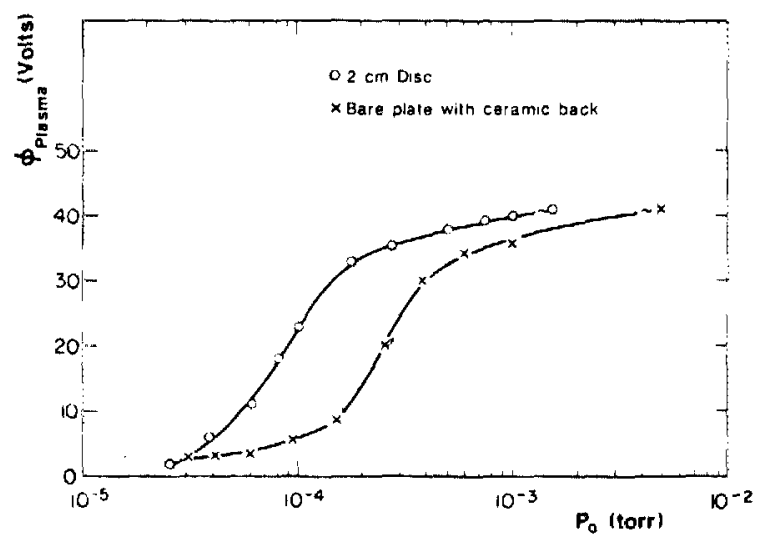

FIG. 9. Plasma potential far from the biased plate vs neutral pressure for plate with ceramic back and plate with $2-\mathrm{cm}$ insulating disk on the front surface. The plate was biased at $+40 \mathrm{~V}$.
Figure 9 also shows the plasma potential versus neutral pressure for a plate with a $2-\mathrm{cm}$ insulating disk on the front with no coating on the back. The relative size of the pumping area in the two cases can be approximated by the ratios of the radii of the insulator, i.e., 3.5:1. Note that the two curves are displaced by approximately 3 .

In summary, we have given evidence for dc pumping in a cold laboratory plasma. Although this is a geometrical effect, it does show the importance of floating objects interacting with positively biased anodes. This was clearly shown by the fingerprint, and emphasizes the need for cleanliness.

\section{ACKNOWLEDGMENTS}

We would like to thank En Yao Wang, Dan Diebold, and Dr. Tom Intrator for many helpful suggestions. This work was supported by NSF Grant No. ECS-8314488.

'R. J. Armstrong and H. Fujita, Proceedings on Second Symposium on Plasma Double Layers, Innsbruck, Austria, edited by $\mathbf{R}$. Schrittwieser (Medieninhaber, Innsbruck, 1984), p. 194.

${ }^{2}$ N. Hershkowitz, G. L. Payne, C. Chan, and J. DeKock, Plasma Phys. 23, 903 (1981).

${ }^{3}$ P. Coakley, L. Johnson, and N. Hershkowitz, Phys. Lett. 70A, 425 (1978).

${ }^{4}$ N. Sato, R. Hatakeyama, S. Iizuka, T. Mieno, K. Saeki, J, Juul Rasmussen, and P. Michelson, Phys. Rev. Lett. 45, 1330 (1981).

${ }^{5}$ H. S. Maciel and J. E. Allen, Proceedings on Second Symposium on Plasma Double Layers, Innsbruck, Austria, edited by R. Schrittwieser (Medieninhaber, Innsbruck, 1984), p. 218.

${ }^{6} \mathrm{M}$. Temerin, K. Arny, W. Lotko, and F. S. Mozer, Phys. Rev. Lett. 48, 1175 (1982).

${ }^{7}$ M. Temerin and F. S. Mozer, Second Symposium on Double Layers and Related Topics, Innsbruck, A ustria, edited by R. Schrittwieser (Medieninhaber, Innsbruck, 1984), p. 119.

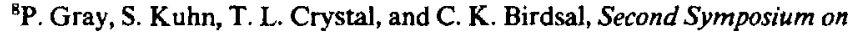
Double Layers and Related Topics, Innsbruck, Austria, edited by R. Schrittwieser (Medieninhaber, Innsbruck, 1984), p. 266.

${ }^{9}$ D. E. Baldwin and B. G. Logan, Phys. Rev. Lett. 43, 1318 (1979).

${ }^{10}$ D. P. Grubb, S. L. Allen, T. A. Casper, J. F. Clauser, F. H. Coensgen, D. L. Correll, W. F. Cummins, C. C. Damm, J. H. Foote, R. K. Goodman, D. N. Hill, E. B. Hooper, Jr., R. S. Hornady, A. L. Hunt, R. G. Kerr, G. W. Lepelmeier, J. Marilleau, J. M. Moller, A. W. Molvik, W. E. Nexsen, W. L. Pickles, G. D. Porter, P. Poulsen, E. H. Selver, T. C. Simonen, B. W. Stallard, W. C. Turner, W. L. Hsu, T. L. Yu, J. D. Barter, T. Christensen, G. Dimonte, T. W. Romesser, R. F. Ellis, R. A. James, G. J. Lasnier, L. V. Berzins, M. R. Carter, C. A. Clower, B. H. Failor, S. Falabella, M. Flammer, and T. Nash, Phys. Rev. Lett. 53, 783 (1984).

'M. Inutake, T. Cho, M. Ichimura, K. Ishii, A. Itakura, K. Katanuma, Y. Kiwamato, Y. Kusama, A. Mase, S. Miyoshi, Y. Nakashima, T. Saito, A. Sakasai, K. Sawada, I. Wakaida, N. Yamaguchi, and K. Yatsu, Phys. Rev. Lett. 55, 939 (1985).

${ }^{12}$ F. Chen, Plasma Diagnostic Techniques, edited by R. H. Huddlestone and S. L. Leonard (Academic, New York, 1965), p. 113.

${ }^{13}$ S. S. Levine and F. W. Crawford, J. Plasma Phys. 23, 223; 24, 359 (1980).

${ }^{14}$ N. Sato, R. Hatakeyama, S. Lizuka, T. Mieno, K. Saeki, J. Rasmussen, and P. Michelson, Phys. Rev. Lett. 45, 1330 (1981).

${ }^{15} \mathrm{H}$. Fujita and S. Yagura, Second Symposium on Plasma Double Layers and Related Topics, Innsbruck, Austria, edited by R. Schrittwieser (Medieninhaber, Innsbruck, 1984), p. 206.

${ }^{16} \mathrm{~T}$. C. Simonen, ed., Summary of TMX-U Results: 1984, Lawrence Livermore National Laboratory Report UCID-20274, pp. 3-97.

${ }^{17}$ N. Hershkowitz, R. Breun, J. Callen, C. Chan, J. Ferron, S. Golovato, J. Pew, B. Nelson, D. Sing, and L. Yujiri, Phys. Rev. Lett. 49, 1489 (1982).

${ }^{18}$ F. H. Coensgen, C. A. Aderson, T. A. Casper, J. F. Clauser, W. C. Condit, D. L. Correl, W. F. Cummins, J. C. Davis, R. P. Drake, J. H. Foote, A. H. Futch, R. K. Goodman, D. P. Grubb, G. A. Hallock, R. S. Hornady, A. L. 
Hunt, B. G. Logan, R. H. Monger, W. E. Nexsen, T. C. Simonen, D. R. Slaughter, B. W. Stallard, and O. T. Strand, Phys. Rev. Lett. 44, 1132 (1980).

${ }^{19} \mathrm{C}$. Chan, T. Intrator, and N. Hershkowitz, Phys. Lett. 91A, 167 (1982).

${ }^{20}$ J. R. Smith, N. Hershkowitz, and P. Coakley, Rev. Sci. Instrum. 50, 210 (1979).
${ }^{2} \mathrm{D}$. Bohm, The Characteristics of Electrical Discharges in Magnetic Fields, edited by R. K. Wakerling and A. Gutherie (McGraw-Hill, New York, 1949), p. 77

${ }^{22}$ D. Rapp and P. Fuglander-Golden, J. Chem. Phys. 43, 1464 (1965).

${ }^{2.3}$ B. M. Smirnov and M. I. Chibisov, Soviet Phys. Tech. Phys. 10, 88 (1965). 